\title{
Lipid and Lipoprotein Profile in HIV-Infected and Non-Infected Diabetic Patients: A Comparative Cross-Sectional Study Design, Southwest Ethiopia
}

\author{
Shiferaw Woyesa' \\ Aklilu Mamo (D) \\ Zeleke Mekonnen (D) \\ Gemeda Abebe \\ Esayas Kebede Gudina $\left(\mathbb{D}^{2}\right.$ \\ Tesfaye Milkesa ${ }^{3}$ \\ 'School of Medical Laboratory Science, \\ Jimma University, Jimma, Ethiopia; \\ ${ }^{2}$ Department of Internal Medicine, Jimma \\ University, Jimma, Ethiopia; ${ }^{3}$ Ambo \\ University Hospital, Ambo, Ethiopia
}

Correspondence: Shiferaw Woyesa

Email bekeleshiferaw@yahoo.com
Background: Lipoproteins are complexes of lipids and proteins that are essential for the transport of cholesterol, triglycerides, and fat-soluble vitamins. The linkage between chronic diseases like diabetes mellitus and HIV infection increases the complication of the diseases and worsens the clinical outcome of the patients.

Purpose: To assess and compare lipid and lipoprotein profiles among HIV-infected and noninfected diabetic patients, and to identify independent predictor variables for abnormal lipid and lipoprotein profiles.

Patients and Methods: A comparative cross-sectional study design was used to carry out the research, and a convenient sampling technique was used to include 96 adult diabetic patients (48 HIV-infected and 48 non-infected diabetics). Socio-demographic and clinical data were collected by interviewer-administered questionnaire. Five milliliter blood sample was collected and processed for lipid and lipoprotein profile measurement. Multivariate and bivariate logistic regressions were used to identify independent predictor variables for abnormal lipid and lipoprotein profiles.

Results: The prevalence of diabetic dyslipidemia was $41.7 \%$ and $37.5 \%$ in $\mathrm{HIV}$-infected and non-infected diabetic patients, respectively. Hypercholesterolemia was more commonly detected among HIV-infected diabetic patients than non-HIV-infected, $25.0 \%$ versus $18.8 \%$, respectively. Similarly, hypertriglyceridemia was more commonly observed in HIVinfected $(31.3 \%)$ than non-infected diabetic patients $(20.8 \%)$. About $25.0 \%$ HIV-infected diabetic patients had combined hyperlipidemia (hypercholesterolemia plus hypertriglyceridemia); and about $4.2 \%$ had hypoalphalipoproteinemia or isolated low HDL-C. Being female and long duration of diabetes mellitus were independent predictor variables for abnormal lipid and lipoprotein profiles in HIV-infected patients. Similarly, being female and high blood pressure were independent predictor variables in non-HIV-infected diabetic patients.

Conclusion: High prevalence lipid and lipoprotein abnormalities were detected in HIVinfected diabetic patients even though the abnormalities were also common in non-HIV comorbid diabetic patients. Hence, proactive screening and treatment of blood glucose, lipid, and lipoprotein abnormalities are critically important and should be part of comprehensive HIV care.

Keywords: diabetes mellitus, hypertriglyceridemia, lipoproteins, hypercholesterolemia, HIV-infection

\section{Introduction}

Lipids occur in all types of cells with great contribution to cellular structure and as the richest source of energy ${ }^{1,2}$; these are chemically heterogeneous group and 
insoluble in water or plasma so that they need carrier proteins or lipoproteins to be transported..$^{3-5}$ Lipoproteins play a cardinal role in transporting hydrophobic lipids, primarily, triglycerides and cholesterol via body fluids from the liver to peripheral tissues and the transport of cholesterol from peripheral tissues to the liver. ${ }^{3,6,7}$ Dietary triglycerides and cholesterol are transported to the periphery and liver by exogenous lipoprotein metabolic pathway, whereas endogenously synthesized hepatic lipids are transported to the periphery by endogenous lipoprotein metabolic pathway that is mediated by very low-density lipoprotein (VLDL) ${ }^{4,8,9}$ The triglycerides of chylomicrons are hydrolyzed by lipoprotein lipase (LPL) releasing free fatty acids that are taken up by adjacent myocytes or adipocytes. ${ }^{3,8}$ Similarly, the triglycerides of VLDL are hydrolyzed by LPL in adipose tissue and muscle producing intermediate density lipoproteins (IDLs) that contain roughly similar amounts of cholesterol and triglyceride. ${ }^{3,4,10}$ About $40-60 \%$ of IDLs are removed by the liver and the remainder is remodeled by hepatic lipase to form low-density lipoprotein (LDL) that contains greater than $50 \%$ of total plasma cholesterol. ${ }^{4,11}$ Another lipoprotein is high-density lipoprotein (HDL) that contains trace amount of triglycerides and significantly useful in transporting excess cholesterol from the plasma membrane of the peripheral cells to liver and intestine. ${ }^{3,12,13}$

Serum cholesterol, particularly the LDL, is one of the most important identified risk factors for coronary heart disease (CHD). High triglyceride is also generally accepted as a significant risk factor. On the other hand, an elevated HDL is considered as protective of CHD. ${ }^{3}$ Sedentary lifestyle, cigarette smoking, advancing age ( $\mathrm{M}>45$ years, $\mathrm{F}>55$ years), hypertension and diabetes mellitus are the major risk factors for CHD. ${ }^{3,4}$ Dyslipidemia is a common characteristic of diabetes mellitus, and there is a link between atherosclerosis and hyperlipidemia in diabetic patients. ${ }^{9,14}$ The risk of CHD is stronger in diabetic patients at any serum cholesterol level with hypertriglyceridemia than in general population. ${ }^{15}$ The linkage between HIV and diabetes mellitus makes the diagnosis and management of these diseases more difficult and also increasing the complications and leading to worsen the clinical outcome of the patients. ${ }^{16,17}$ Co-morbid medical conditions including diabetes mellitus, dyslipidemia and other metabolic disorders are challenging in managing people living with HIV and diabetes mellitus. ${ }^{9,18-25}$ Like other general populations, HIV-infected individuals are exposed to different socioeconomic and cultural factors, including sedentary lifestyle and unhealthy dietary practices. Such problems are highly prevalent in low-income countries. ${ }^{26}$ Diabetes and other metabolic abnormalities among HIVinfected individuals are not well studied in low-income countries including Ethiopia where the availability of health-care resources is limiting. Therefore, the objective of this study was to assess and determine lipid and lipoprotein profile in HIV-infected diabetic patients and to compare the findings with HIV-non-infected diabetic patients.

\section{Materials and Methods Study Objective, Period and Setting}

The study was conducted in Jimma Zone public health institutions from February 05, 2019, to June 28, 2019. Jimma zone is located in Oromia Regional State southwest Ethiopia. Jimma Zone has one tertiary hospital, two general hospitals and four primary hospitals. The Jimma University Medical Center is the only teaching and referral hospital in southwest Ethiopia with about 800 beds capacity and over 20 million catchment populations.

\section{Study Design, Sample Size Determination and Sampling Technique}

A comparative cross-sectional study design was used to carry out the study. The sample size was calculated by using a double population proportion formula, $\mathrm{n}=\left(\mathrm{Z}_{\alpha / 2}\right.$ $\left.+\mathrm{Z}_{\beta}\right)^{2} *\left(\mathrm{p}_{1}\left(1-\mathrm{p}_{1}\right)+\mathrm{p}_{2}\left(1-\mathrm{p}_{2}\right)\right) /\left(\mathrm{p}_{1}-\mathrm{p}_{2}\right)^{2}$, by considering $\mathrm{P}_{1}$ $=0.127^{34}$ for HIV-infected diabetic patients and $\mathrm{P}_{2}$ $=0.385^{35}$ for non-HIV-infected diabetic patients with $95 \%$ confidence interval and $80 \%$ power. A convenient sampling technique was used to include 96 adult diabetic patients (48 HIV-infected and 46 non-HIV-infected). Severely ill and individuals on lipid profile-lowering drugs were excluded from the study as it severely interfere the lipid profile result.

\section{Data Collection and Blood Chemistry}

Socio-demographic and clinical data were collected by trained data collectors using an interviewer-administered questionnaire. Anthropometric data and blood pressure measurements were performed by clinical nurses working in chronic illness clinic according to World Health Organization (WHO) guidelines. ${ }^{27}$

The body mass index (BMI) was calculated as weight/ height square expressed as $\mathrm{kg} / \mathrm{m}^{2}$. Study subjects with BMI value of $25.0-29.99 \mathrm{~kg} / \mathrm{m}^{2}$ were categorized as 
overweight and those with $\geq 30.0 \mathrm{~kg} / \mathrm{m}^{2}$ as obese. Blood pressure values of $\geq 140 \mathrm{mmHg}$ for systolic and/ or $\geq 90$ $\mathrm{mmHg}$ for diastolic were categorized high blood pressure or hypertension. ${ }^{28}$

About 5-milliter blood sample was collected from each study subject and processed based on the standard operating procedure for sample processing. After serum had been separated from whole blood cell, the samples were analyzed for lipid parameter (triglyceride and total cholesterol), and lipoproteins (high-density lipoprotein cholesterol and low-density lipoprotein cholesterol) by using Horiba ABX SAS (Montpellier, France) chemistry analyzer by strictly applying the basic test principle for each lipid and lipoprotein parameter.

The lipid profile and lipoprotein serum results were interpreted based on WHO dyslipidemia guideline, as hypercholesterolemia: if serum total cholesterol level $>160 \mathrm{mg} / \mathrm{dl}$; hypertriglyceridemia: if serum triglyceride level $\geq 200 \mathrm{mg} / \mathrm{dl}$; elevated low-density lipoprotein cholesterol: if serum LDL-C level $\geq 160 \mathrm{mg} / \mathrm{dl}$; low high-density lipoprotein cholesterol:-if HDL-C $<40 \mathrm{mg} / \mathrm{dl}$ for male and $<30 \mathrm{mg} / \mathrm{dl}$ for female. ${ }^{5}$

\section{Statistical Analysis}

After proper cleaning for completeness and consistency, the data were entered into the Epi-data version 3.1 and later exported to SPSS version 21.0 for analysis and interpretation. Descriptive statistics were used to explain data in numbers and proportions. Binary and multivariate logistic regression analyses were used to assess the relationship between independent and predictor variables. $\mathrm{P}$ value $<0.05$ was used as a threshold level for statistical significance.

\section{Results}

\section{Socio-Demographic and Clinical Characteristics of Study Subjects}

A total of 96 (48 HIV-infected and 48 non-HIV-infected) diabetic patients were included in the study with the mean \pm SD age of $51.6 \pm 10$ years for HIV-infected and 48. $8 \pm 9.8$ years for non-HIV-infected diabetic patients; age range of 30-70 and 28-66 years for HIV-infected and non-infected study subjects, respectively. The proportion of male study participants were higher in HIV-infected diabetic patients as compared to non-HIV-infected ( $75 \%$ versus $47.9 \%$ respectively). The majority of the study subjects, $87.5 \%$ for HIV-infected and $81.3 \%$ for non-HIV-infected, belonged to the age group of 40 years or older. About $29.2 \%$ and $10.4 \%$ HIV-infected patients were overweight and obese, respectively. Concerning duration of diabetes mellitus, majority of HIV-infected diabetic patients, $60.4 \%$, were less than 5 years, whereas about $58.3 \%$ of non-HIV-infected diabetic patients were greater or equal to 5 years. Almost comparable proportion of study groups had been taking oral glucose lowering agents; $56.3 \%$ and $52.1 \%$ HIV-infected and non-infected diabetic patients, respectively. About $64.6 \%$ and $70.8 \%$ HIV-infected diabetic patients were on a TDF+3TC+EFV drug regimen and had CD4 count greater or equal to 500 cells $/ \mathrm{mm}^{3}$, respectively. Concerning substance use, about $29.2 \%$ and $18.8 \%$ of HIV-infected and non-infected diabetics, respectively, had the habit of cigarette smoking during the study period (Table 1).

The prevalence of diabetic dyslipidemia was $41.7 \%$ and $37.5 \%$ in HIV-infected and non-infected diabetic patients, respectively. About 25.0\% HIV-infected diabetic patients had high total cholesterol level as compared to $18.8 \%$ among non-HIV-infected diabetic patients. Similarly, higher rate of hypertriglyceridemia was observed among HIV-infected than non-HIV-infected diabetic patients $(31.3 \%$ versus $20.8 \%$, respectively). The serum abnormality of APO-B containing lipoprotein that carries about $50 \%$ of total cholesterol or LDL-C was about $35.4 \%$ in HIV-infected and $29.2 \%$ in non-HIV-infected diabetic patients. Again, the low level of non-APO-B containing lipoprotein or HDL-C was more common in HIV-infected diabetic patients than non-HIV-infected ( $29.2 \%$ versus $18.8 \%$, respectively). From the total HIVinfected diabetic patients about $25.0 \%$ and $4.2 \%$ had combined hyperlipidemia and hypoalphalipoproteinemia respectively showing increased serum level of total cholesterol and triglyceride together, and isolated low level of HDL-C (Table 2).

The multivariate logistic regression analysis revealed that being female (AOR: 6.30, 95\% C.I: $1.398-28.390$, P: 0.0165 ) and long duration of diabetes ( $\geq 5$ years) (AOR: 4.93, 95\% C.I: 1.091-22.297, P: 0.038) were independently associated with abnormal lipid and lipoprotein profile in HIV-infected diabetic patients. Similarly, being female (AOR: 9.23, 95\% C.I: 1.661-51.308, P: 0.011) and high systolic blood pressure (AOR: 3.92, C.I: 1.012 15.214, P: 0.048); high diastolic blood pressure (AOR: 13.04, 95\% C.I: 1.804-94.25, P: 0.011) were independent predictor variables for abnormal lipid and lipoprotein profile in non-HIV-infected diabetic patients. Being female 
Table I Socio-Demographic and Clinical Characteristics of HIV-Infected and Non-Infected Diabetic Patients

\begin{tabular}{|c|c|c|c|c|}
\hline Variable & Category & HIV-Infected, N(\%) & HIV-Non-Infected, N(\%) & Total, N(\%) \\
\hline \multirow[t]{2}{*}{ Sex } & Male & $36(75.0)$ & $23(47.9)$ & $59(61.5)$ \\
\hline & Female & $12(25.0)$ & $25(52.1)$ & $37(38.5)$ \\
\hline \multirow[t]{2}{*}{ Age (years) } & $18-39$ & $6(12.5)$ & $9(18.8)$ & $15(15.6)$ \\
\hline & $\geq 40$ & $42(87.5)$ & $39(81.3)$ & $81(84.4)$ \\
\hline \multirow[t]{2}{*}{ Marital status } & Married & $39(81.3)$ & $38(79.2)$ & $77(80.2)$ \\
\hline & Others & $9(18.8)$ & $10(20.8)$ & $19(19.8)$ \\
\hline \multirow[t]{2}{*}{ Occupation } & Employed & $25(52.1)$ & $22(45.8)$ & $47(48.9)$ \\
\hline & Unemployed & $23(47.9)$ & $26(54.2)$ & $49(51.04)$ \\
\hline \multirow[t]{2}{*}{ Residence } & Urban & $31(64.6)$ & $35(72.9)$ & $66(68.8)$ \\
\hline & Rural & $17(35.4)$ & $13(27.1)$ & $30(32.3)$ \\
\hline \multirow[t]{2}{*}{ Educational status } & No formal education & $6(12.5)$ & $7(14.6)$ & $13(13.5)$ \\
\hline & Formal education & $42(87.5)$ & $4 \mathrm{I}(85.4)$ & $83(86.5)$ \\
\hline \multirow[t]{3}{*}{ BMI $\left(\mathrm{kg} / \mathrm{m}^{2}\right)$} & Normal & $29(60.4)$ & $39(81.3)$ & $68(70.8)$ \\
\hline & Overweight & $14(29.2)$ & $7(14.6)$ & $21(21.9)$ \\
\hline & Obesity & $5(10.4)$ & $2(4.2)$ & $7(7.3)$ \\
\hline \multirow[t]{2}{*}{ Systolic blood pressure } & Normal (<140mmHg) & $25(52.1)$ & $30(62.5)$ & $55(57.3)$ \\
\hline & Abnormal $(\geq \mid 40 \mathrm{mmHg})$ & $23(47.9)$ & $18(37.5)$ & $4 I(42.7)$ \\
\hline \multirow[t]{2}{*}{ Diastolic blood pressure } & Normal $(<90 \mathrm{mmHg})$ & $23(47.9)$ & $15(31.3)$ & $38(39.6)$ \\
\hline & Abnormal $(\geq 90 \mathrm{mmHg})$ & $27(56.3)$ & $33(68.8)$ & $60(62.5)$ \\
\hline \multirow[t]{2}{*}{ Duration DM } & $<5$ years & $29(60.4)$ & $20(41.7)$ & $49(51.0)$ \\
\hline & $\geq 5$ years & 19(39.6) & $28(58.3)$ & $47(49.0)$ \\
\hline \multirow[t]{3}{*}{ Anti-hyperglycemia } & Insulin & $19(39.6)$ & $15(3 \mid .3)$ & $34(35.4)$ \\
\hline & Metformin & $27(56.3)$ & $25(52.1)$ & $52(54.2)$ \\
\hline & Others & $2(4.2)$ & $8(16.7)$ & $10(10.4)$ \\
\hline \multirow[t]{4}{*}{ ART drug regimen } & $\mathrm{TDF}+3 \mathrm{TC}+\mathrm{EFV}$ & $31(64.6)$ & - & $31(64.6)$ \\
\hline & $\mathrm{AZT}+3 \mathrm{TC}+\mathrm{EFV}$ & $14(29.2)$ & - & $14(29.2)$ \\
\hline & $A B C+3 T C+E F V$ & $2(4.2)$ & - & $2(4.2)$ \\
\hline & Other & $\mathrm{I}(2.1)$ & - & $I(2.1)$ \\
\hline \multirow[t]{2}{*}{ CD4 count (in cells $/ \mathrm{mm}^{3}$ ) } & $<500$ & $14(29.2)$ & - & $14(29.2)$ \\
\hline & $\geq 500$ & $34(70.8)$ & - & $34(70.8)$ \\
\hline \multirow[t]{2}{*}{ Smoking } & Yes & $14(29.2)$ & $9(18.8)$ & $23(24.0)$ \\
\hline & No & $34(70.8)$ & $29(60.4)$ & $63(65.6)$ \\
\hline \multirow[t]{2}{*}{ Khat chewing } & Yes & $7(14.6)$ & II(22.9) & $18(18.8)$ \\
\hline & No & $4 I(85.4)$ & $27(56.3)$ & $68(70.8)$ \\
\hline
\end{tabular}

Abbreviations: ART, anti-retroviral therapy; BMI, body mass index; $\mathrm{mmHg}$, millimeter of mercury; ABC, abacavir; AZT, zidovudine; EFV, efavirenz; 3TC, lamivudine; TDF, tenofovir disoproxil fumarate. 
Table 2 Dyslipidemia and Lipoprotein and Lipid Profile in HIV-Infected and Non-Infected Diabetic Patients

\begin{tabular}{|c|c|c|c|c|}
\hline Variable & Category & $\begin{array}{l}\text { HIV-Infected DM, } \\
\text { N(\%) }\end{array}$ & $\begin{array}{l}\text { Non-HIV-Infected DM, } \\
\text { N(\%) }\end{array}$ & Total, N(\%) \\
\hline \multirow[t]{2}{*}{ Dyslipidemia } & Yes & $20(41.7)$ & $18(37.5)$ & $38(39.6)$ \\
\hline & No & $28(58.3)$ & $30(62.5)$ & $58(60.4)$ \\
\hline \multirow[t]{2}{*}{ Total cholesterol } & Elevated & $12(25.0)$ & $9(18.8)$ & $2 \mathrm{I}(2 \mathrm{I} .8)$ \\
\hline & Normal & $36(75.0)$ & $39(81.3)$ & $75(78.1)$ \\
\hline \multirow[t]{2}{*}{ Triglyceride } & Elevated & $15(31.3)$ & $10(20.8)$ & $25(26.0)$ \\
\hline & Normal & $33(68.8)$ & $38(79.2)$ & $7 \mid(74.0)$ \\
\hline \multirow[t]{2}{*}{ LDL-C } & Elevated & $17(35.4)$ & $14(29.2)$ & $3 I(32.3)$ \\
\hline & Normal & $3 I(64.6)$ & $34(70.8)$ & $65(67.7)$ \\
\hline \multirow[t]{2}{*}{ HDL-C } & Low & $14(29.2)$ & $9(18.8)$ & $23(24.0)$ \\
\hline & Elevated & $34(70.8)$ & $39(8 \mid .3)$ & $73(76.0)$ \\
\hline \multirow[t]{2}{*}{ Combined hyperlipidemia } & Yes & $12(25.0)$ & $9(18.8)$ & $2 I(2 I .9)$ \\
\hline & No & $3(6.3)$ & $I(2 . I)$ & $4(8.3)$ \\
\hline \multirow[t]{2}{*}{ Hypoalphalipoproteinemia (HDL-C <38mg/dl) } & Yes & $2(4.2)$ & $0(0.0)$ & $2(4.2)$ \\
\hline & No & $12(25.0)$ & $9(18.8)$ & $21(21.9)$ \\
\hline
\end{tabular}

Abbreviations: LDL-C, low-density lipoprotein-cholesterol; HDL-C, high-density lipoprotein-cholesterol.

was about 6.3 times more likely to develop dyslipidemia than male in HIV-infected diabetic patients. The odds of developing dyslipidemia among HIV-infected diabetic patients with abnormal systolic blood pressure was about 3.53 times more likely than those with normal systolic blood pressure. HIV-infected diabetic patients with longer duration of diabetes mellitus ( $\geq 5$-years) were about 4.93 times more likely to develop dyslipidemia as compared to those with less duration of diabetes mellitus ( $<5$ years) (Table 3).

\section{Discussion}

The total prevalence of dyslipidemia was $39.6 \%$, and it was a little bit more common in HIV-infected diabetic patients (41.7\%) as compared to non-HIV-infected diabetic patients (37.5\%). Comparable prevalence of dyslipidemia was reported from Khartoum with the range of $13-70 \%$ diabetic dyslipidemia in HIV-infected patients. ${ }^{29}$ About $21.8 \%$ diabetic patients were hypercholesterolemic, of which about $25.0 \%$ and $18.8 \%$ were from HIV-infected and non-infected diabetic study groups, respectively. HIV-infected diabetic study subjects were more hypertriglyceridemic than the HIV-non-infected study subjects, $31.3 \%$ versus $20.8 \%$ respectively and the total hypertriglyceridemia was about
26.0\%. Similar findings in which HIV-infected diabetic patients were more hypercholesterolemic and hypertriglyceridemic than non-HIV-infected diabetic patients were reported from Massachusetts. ${ }^{6}$ In contrast to this, the study reported from Australia showed that there was no difference in serum concentration of triglyceride in HIV-infected and non-infected diabetic patients. ${ }^{30}$ Some study subjects had both elevated cholesterol and triglyceride together, which are considered to be risk factors for coronary heart disease (CHD), and thus about $25.0 \%$ HIV-infected and $18.8 \%$ noninfected diabetic patients had combined hyperlipidemia. Elevation of LDL-C is considered as an established risk factor for CHD and the elevation of this lipoprotein was more common among HIV-infected diabetic patients than non-infected, $35.4 \%$ versus $29.2 \%$, and a total of $32.3 \%$ study subjects had abnormal level of this lipoprotein. The highest concentration of HDL-C is very helpful in maintaining the equilibrium of cholesterol in peripheral cells by removing excess cholesterol via the reverse cholesterol transport pathway. Thus, its high blood concentration has anti-atherogenic property, whereas its low concentration is considered to be an independent risk factor for atherosclerosis. A majority, $70.8 \%$ and $81.3 \%$, of HIV-infected and noninfected diabetic patients, respectively, had the highest 
Table 3 Multivariate Logistic Regression Analysis in HIV-Infected and Non-Infected Diabetics

\begin{tabular}{|c|c|c|c|c|c|}
\hline \multirow[t]{2}{*}{ Variable } & \multirow[t]{2}{*}{ Category } & \multicolumn{2}{|l|}{ HIV-Infected } & \multicolumn{2}{|l|}{ HIV-Non-Infected } \\
\hline & & AOR(95\% Cl) & $P$ value & AOR(95\% Cl) & $P$ value \\
\hline \multirow[t]{2}{*}{ Sex } & Male & $0.16(0.035-0.715)$ & I & $0.11(0.019-0.602)$ & I \\
\hline & Female & $6.30(1.398-28.390)$ & 0.0165 & $9.23(I .66 I-5 I .308)$ & 0.011 \\
\hline \multirow[t]{2}{*}{ Systolic BP (mmHg) } & Normal & $0.28(0.063-1.266)$ & 1 & $0.26(0.066-0.988)$ & I \\
\hline & Abnormal & $3.53(0.790-15.775)$ & 0.099 & $3.92(1.012-15.214)$ & 0.048 \\
\hline \multirow[t]{2}{*}{ Diastolic (mmHg) } & Normal & $0.35(0.098-1.233)$ & 1 & $0.08(0.011-0.554)$ & 1 \\
\hline & Abnormal & $2.87(0.8 \mid I-10.177)$ & 0.102 & |3.04(I.804-94.25) & 0.011 \\
\hline \multirow[t]{2}{*}{ Alcohol use } & Yes & $4.10(0.898-18.722)$ & 0.07 & $2.82(0.758-10.50 I)$ & 0.122 \\
\hline & No & $0.24(0.053-1.113)$ & 1 & $0.36(0.095-1.320)$ & 1 \\
\hline \multirow[t]{2}{*}{ Khat chewing } & Yes & $4.72(0.763-29.248)$ & 0.10 & I.54(0.393-6.026) & 0.536 \\
\hline & No & $0.212(0.034-1.311)$ & I & $0.65(0.166-2.546)$ & I \\
\hline \multirow[t]{2}{*}{ Duration of diabetes mellitus } & $<5$ years & $0.20(0.045-0.917)$ & 1 & $0.42(0.08-2.285)$ & 1 \\
\hline & $\geq 5$ years & $4.93(1.091-22.297)$ & 0.038 & $2.36(0.438-|2.77|)$ & 0.317 \\
\hline
\end{tabular}

Abbreviations: AOR, adjusted odds ratio; BP, blood pressure; $\mathrm{mmHg}$, millimeter of mercury; HIV, human immunodeficiency virus.

concentration of HDL-C although 29.2\% HIV-infected and $18.8 \%$ non-HIV-infected diabetic patients had low serum concentration of HDL-C. This finding is comparable with the finding reported from Tunisia in which HDL-C value in selected study subjects was $26.6 \%{ }^{31}$ From the total HIVinfected diabetic patients with low concentration of HDL-C, $4.2 \%$ study subjects had an isolated decrease in HDL-C or hypoalphalipoproteinemia, which might be due to mutation of apolipoprotein A-I. This finding is almost comparable to the finding reported from Japan with 6.0\%hypoalphalipoproteinemia, ${ }^{32}$ but much less than the finding from Indonesia with $30 \%$ hypoalphalipoproteinemia. ${ }^{33}$ Even though the BMI measurement was abnormal among non-HIV-infected diabetic patients, the prevalence of abnormal BMI was twice among the co-morbidities.

The multivariate analysis revealed that being female and long duration of diabetes were independent risk factors for hyper- and hypolipoproteinemia, and hyperlipidemia in HIV-infected diabetic patients. Similarly, being female and abnormal diastolic blood pressure were independent predictor variables for the same abnormality in non-HIV-infected diabetic patients. The non-HIV-infected diabetic patients with abnormal diastolic blood pressure had about 13 times more likely to develop dyslipidemia than those with normal diastolic blood pressure. Similarly, female non-HIV-infected diabetic patients had more than nine times more likely to have abnormal lipoproteins and hyperlipidemia.

This shows that HIV infection is likely to worsen metabolic abnormalities among diabetic patients. In addition to the co-morbidities, social determinants of health include access to healthy foods, quality of health care access; and economic stability to choice and pay for quality of medical care might contribute to higher prevalence of serum lipid and lipoprotein abnormality in diabetic patients.

\section{Limitation of the Study}

The sample size was small even though we used all HIVinfected diabetic patients in the study area. Due to the impact from cross-sectional study, design we could not determine whether the exposure or outcome came first.

\section{Conclusion}

More lipid and lipoprotein abnormality was detected in HIVinfected diabetic patients, although the abnormality was common in non-HIV co-morbid diabetes mellitus. Being female and long duration of diabetes mellitus were independent predictor variables for abnormal lipid and lipoprotein profile in HIV-infected and HIV-negative diabetic patients. Thus, appropriate diagnosis and management of lipoprotein disorders is critically important and lipid abnormalities 
should be assessed aggressively and treated as part of comprehensive diabetic care. Hence, proactive screening and treatment of blood glucose, lipid, and lipoprotein abnormalities should be part of comprehensive HIV care.

\section{Abbreviations}

ABC, abacavir; AOR, adjusted odds ratio; ART, antiretroviral therapy; AZT, azidothymidine; BMI, body mass index; BP, blood pressure; CHD, coronary heart disease; CD4, cluster of differentiation; EFV, efavirenz; HDL-C, high-density lipoprotein-cholesterol; HIV, human immunodeficiency virus; LDL-C, low-density lipoproteincholesterol; $\mathrm{mmHg}$, millimeter of mercury; 3TC, lamivudine; TDF, tenofovir disoproxil fumarate; WHO, World Health Organization.

\section{Data Sharing Statement}

Data are available from the corresponding author on reasonable request.

\section{Ethical Clearance and Consent to Participant}

The study has been conducted in accordance with the principles stated in the Declaration of Helsinki, and thus an ethical approval had been taken from the Institutional Review Board of Jimma University Institute of Health. Official permission was also obtained from Jimma Zone Health Office and heads of each hospital involved. Additionally, written informed consent was obtained from all study participants. Confidentiality of the collected data was ensured through anonymity.

\section{Acknowledgments}

We would like to thank study participants for their voluntarily participation, and Jimma University for financing the study. Our great thanks also go to those who made any contribution to the successful accomplishment of this study directly or indirectly.

\section{Author Contributions}

All authors contributed to data analysis, drafting or revising the article, have agreed on the journal to which the article will be submitted, gave final approval of the version to be published, and agree to be accountable for all aspects of the work.

\section{Funding}

This research project had been funded by Jimma University.

\section{Disclosure}

The authors reported there is no financial or any other conflict of interest.

\section{References}

1. Gurr MI, Harwood JL, Frayn KN. Lipid Biochemistry. 5th ed. Blackwell science; 2002.

2. Harvey RA. Lippincott's Illustrated Reviews: Biochemistry. 5th ed. Philadelphia; 2011.

3. Bishop ML. Clinical Chemistry Principles, Techniques, and Correlations. 8th ed. Wolters Kluwer; 2018.

4. Mcpherson RA, Pincus MR. HENRY'S Clinical Diagnosis and Management by Laboratory Methods. 22nd ed. ELSEVIER SAUNDERS; 2011.

5. Wu L, Parhofer KG. Diabetic dyslipidemia. Metabolism. 2014;63 (12):1469-1479. doi:10.1016/j.metabol.2014.08.010

6. Hadigan C, Meigs JB, Corcoran C, et al. Metabolic abnormalities and cardiovascular disease risk factors in adults with human immunodeficiency virus infection and lipodystrophy. Clin Infect Dis. 2001;32:130-139. doi:10.1086/317541

7. Lake JE, Currier JS. Metabolic disease in HIV infection. Lancet Infect Dis. 2013;13(11):964-975. doi:10.1016/S1473-3099(13) 70271-8

8. Vasudevan DM, Sreekumari S, Kannan V. Textbook of Biochemistry for Medical Students. India: Jaypee Brothers Medical Publishers. Sixth ed. 2011.

9. Dube M, Fenton M. Lipid abnormalities. Clin Infect Dis. 2003;36 (Suppl2):79-83.

10. Jameson JL. HARRISON'S Endocrinology. 3rd ed. McGraw-Hill Education; 2013.

11. Femlak M, Gluba-brzózka A, Cia A, Rysz J. The role and function of HDL in patients with diabetes mellitus and the related cardiovascular risk. Lipids Health Dis. 2017;16:1-9.

12. WHO Guidelines. Prevention of Cardiovascular Disease. WHO; 2007:1-94.

13. Bale P, Oyewo TA. Metabolic control and determinants among HIV-infected type 2 diabetes mellitus patients attending a tertiary clinic in Botswana. Diabetes Metabol Syndr Obes. 2021;14:85.

14. Schofield JD, Liu Y, Rayaz PR, Malik RA, Soran H. Diabetes dyslipidemia. Diabetes Ther. 2016;7(2):203-219. doi:10.1007/ s13300-016-0167-x

15. Waters DD, Hsue PY. Lipid abnormalities in persons living with HIV infection. Can J Cardiol. 2019;35(3):249-259. doi:10.1016/j. cjca.2018.11.005

16. Van Ness SE, Chandra A, Sarkar S, et al. Predictors of delayed care seeking for tuberculosis in southern India: an observational study. BMC Infect Dis. 2017;17(1):1-6. doi:10.1186/s12879-017-2629-9

17. Di Gennaro F, Marotta C, Antunes M, Pizzol D. Diabetes in active tuberculosis in low-income countries: to test or to take care? Lancet Glob Health. 2019;7(6):e707. doi:10.1016/S2214-109X(19)30173-1

18. Ahmed MH, Ahmed MH. Managing dyslipidemia in HIV/AIDS patients: challenges and solutions. HIV/AIDS. 2015;7:1-10. doi:10.2147/HIV.S46028

19. Tadewos A, Addis Z, Ambachew H, Banerjee S. Prevalence of dyslipidemia among HIV-infected patients using first-line highly active antiretroviral therapy in Southern Ethiopia: a cross-sectional comparative group study. AIDS Res Ther. 2012;9:1-8. 
20. Moyo D, Hons B, Tanthuma G, et al. Diabetes mellitus in HIV-infected patients receiving antiretroviral therapy. South Afri Med J. 2014;104(1):37-39.

21. Ataro Z, Ashenafi W, Fayera J, Abdosh T. Magnitude and associated factors of diabetes mellitus and hypertension among adult HIV positive individuals receiving highly active antiretroviral therapy at Jugal Hospital, Harar. HIV/AIDS. 2018;10:181-192.

22. Omech B, Sempa J, Castelnuovo B, et al. Prevalence of HIV-associated metabolic abnormalities among patients taking first-line antiretroviral therapy in Uganda. ISRN AIDS. 2012;2012. doi: $10.5402 / 2012 / 960178$

23. Monroe AK, Glesby MJ, Brown TT. Diagnosing and managing diabetes in HIV-infected patients: current concepts. Clin Infect Dis. 2015;60:453-462. doi:10.1093/cid/ciu779

24. Coelho AR, Moreira FA, Santos AC, et al. Diabetes mellitus in HIVinfected patients: fasting glucose, A1c, or oral glucose tolerance test - which method to choose for the diagnosis? Toxicol Appl Pharmacol. 2018;348:1-13. doi:10.1016/j.taap.2018.04.009

25. Fiseha T, Belete AG, Ishii T, Watanabe M, Noutoshi Y. Diabetes mellitus and its associated factors among human immunodeficiency virus - infected patients on anti - retroviral therapy in Northeast Ethiopia. BMC Res Notes. 2019;12:1-7. doi:10.1186/s13104-0184038-6

26. Patel P, Rose CE, Collins PY, et al. Noncommunicable diseases among HIV-infected persons in low-income and middle-income countries: a systematic review and meta-analysis. Aids. 2018;32 (Suppl 1):S5-20. doi:10.1097/QAD.0000000000001888

27. WHO. Guide to Anthropometry: A Practical Tool for Program Planners, Managers, and Implementers. WHO; 2018:1-30.
28. National Center for Health Statistics. Anthropometry Procedures Manual. National Health and Nutrition examination Survey. National Center for Health Statistics; 2017:1-26.

29. Noor SK, Mital D, Elmadhoun WM. Diabetes, metabolic syndrome and dyslipidemia in people living with HIV in Africa: re-emerging challenges not to be forgotten. HIV/AIDS. 2017;9:193-202. doi:10.2147/HIV.S137974

30. Low H, Hoang A, Pushkarsky T, et al. HIV disease, metabolic dysfunction and atherosclerosis: a three year prospective study. PloS One. 2019;14:1-19.

31. Ben CF, El AJ, Doggui R, Ati-hellal ME. Prevalence of high HDL cholesterol and its associated factors among Tunisian women of childbearing age: a cross-sectional study. Front Oncol. 2021;11. doi:10.3389/fonc.2021.688200

32. Yamakawa-kobayashi K, Yanagi H, Fukayama H, et al. Frequent occurrence of hypoalphalipoproteinemia due to mutant apolipoprotein A-I gene in the population: a population-based survey. Human Mol Genet. 1999;8(2):331-336. doi:10.1093/hmg/8.2.331

33. Kalim H. Hypoalphalipoproteinemia: prevalence and the impact of treatment on reaching HDL cholesterol target level in patients with dyslipidemia. Med J Indones. 2001;10(2):98-102.

34. Mohammed AE, Shenkute TY, Gebisa WC. Diabetes mellitus and risk factors in human immunodeficiency virus-infected individuals at Jimma University Specialized Hospital, Southwest Ethiopia. Diabetes Metab Syndr Obes. 2015;8: 197-206.

35. Ambachew H, Shimelis T, Lemma K. Dyslipidemia among diabetic patients in Southern Ethiopia: Cross-sectional study. Journal of Diabetes and Endocrinology. 2015;6(4):19-24.
HIV/AIDS - Research and Palliative Care

\section{Publish your work in this journal}

HIV/AIDS - Research and Palliative Care is an international, peerreviewed open-access journal focusing on advances in research in HIV, its clinical progression and management options including antiviral treatment, palliative care and public healthcare policies to

\section{Dovepress}

control viral spread. The manuscript management system is completely online and includes a very quick and fair peer-review system, which is all easy to use. Visit http://www.dovepress.com/testimonials.php to read real quotes from published authors. 